\author{
Ecología
}

\title{
Uso de hábitat de dos ecotipos de toninas (Tursiops truncatus) en el golfo de California, México
}

\author{
Habitat use of two ecotypes of Bottlenose dolphins (Tursiops truncatus) in the \\ Gulf of California, Mexico \\ Daniel Guevara-Aguirre ${ }^{a, *}$ y Juan Pablo Gallo-Reynoso ${ }^{b}$ \\ ${ }^{a}$ Posgrado de Ciencias del Mar y Limnología, Instituto de Ciencias del Mar y Limnología, Universidad Nacional Autónoma de México, Circuito Exterior s/n, \\ Ciudad Universitaria, 04510, Ciudad de México, México \\ ${ }^{\mathrm{b}}$ Laboratorio de Ecofisiología, Centro de Investigación en Alimentación y Desarrollo, Carretera al Varadero Nacional km 6.6, 85480, Guaymas, Sonora, México \\ Recibido el 25 de febrero de 2016; aceptado el 24 de mayo de 2016 \\ Disponible en Internet el 21 de agosto de 2016
}

\section{Resumen}

En el golfo de California se han descrito 2 ecotipos de toninas (costero y oceánico) y la mayoría de investigaciones son de regiones costeras. Este estudio analiza si 10 variables ambientales (profundidad, color del mar, visibilidad, estado del mar, salinidad, temperatura superficial, concentración de clorofila, distancia a la costa, pendiente, densidad del agua) determinan el hábitat de estos ecotipos, probando la hipótesis del hábitat local. Se realizaron 13 muestreos costeros y 16 muestreos oceánicos en la cuenca de Guaymas (septiembre 2013-marzo 2014). El análisis de correspondencia indicó que el ecotipo costero se alimenta y transita por la mañana, socializa al medio día y descansa por la tarde; 6 variables (temperatura superficial, visibilidad, salinidad, color del mar, distancia a la costa, pendiente) determinan estos comportamientos. El ecotipo oceánico se alimenta por la mañana y la tarde, socializa al medio día, descansa en la tarde, transita por la mañana y la tarde; 4 variables (pendiente, color del mar, temperatura superficial y profundidad) determinan estos comportamientos. Los GLM indican que 5 variables caracterizan el hábitat de ambos ecotipos (pendiente, concentración de clorofila, distancia a la costa, estado del mar, color del mar). Se comprueba la hipótesis del hábitat local, porque cada ecotipo depende del conjunto de variables de su hábitat.

Derechos Reservados (C) 2016 Universidad Nacional Autónoma de México, Instituto de Biología. Este es un artículo de acceso abierto distribuido bajo los términos de la Licencia Creative Commons CC BY-NC-ND 4.0.

Palabras clave: Nicho ecológico; Modelo lineal generalizado; Análisis de correspondencia

\section{Abstract}

Two ecotypes (coastal and oceanic) of bottlenose dolphins are described in the Gulf of California where the majority of the investigations have been done in coastal regions. This study analyzes if 10 environmental variables (depth, sea color, visibility, sea state, salinity, sea surface temperature, chlorophyll concentration, distance to coast, slope and water density) in Guaymas Basin could differentiate the habitat of both ecotypes, testing the hypothesis of local habitat. Thirteen surveys were conducted along the coastal area and 16 in the offshore area (September 2013-March 2014). Correspondence analysis showed that the coastal ecotype forages and transits in the morning, socializes at noon and rests in the evening, these behaviors were determined by 6 variables (sea surface temperature, visibility, salinity, sea color, distance to coast, and slope). The offshore ecotype forages in the morning and in the afternoon, has a social life at noon, rests in the evening and transits in the morning and afternoon, these behaviors are determined by 4 variables (depth, sea color, sea surface temperature, slope). Ecological niche models showed that 5 variables characterize and differentiate the ecotypes (slope, chlorophyll concentration, distance from the coast, sea state, and sea color). The local habitat hypothesis is proven as each ecotype depends on the cluster of variables of their habitat.

All Rights Reserved () 2016 Universidad Nacional Autónoma de México, Instituto de Biología. This is an open access item distributed under the Creative Commons CC License BY-NC-ND 4.0.

Keywords: Ecological niche; Generalized linear model; Correspondence analysis

\footnotetext{
* Autor para correspondencia.

Correo electrónico: danyguevara47@ hotmail.com (D. Guevara-Aguirre).

La revisión por pares es responsabilidad de la Universidad Nacional Autónoma de México.
} 


\section{Introducción}

El uso del hábitat se refiere a cómo algunas áreas con ciertos factores ecológicos y variables ambientales específicas son utilizadas por diferentes especies afectando su distribución y su abundancia en el ambiente que los rodea (Ferrero, Hobbies y van Blaricom, 2002). En general, estos hábitats se conforman de una gama de microhábitats que difieren de manera física y biológica. Algunos microhábitats proporcionan grandes concentraciones de comida y otros funcionan como barreras contra posibles depredadores (Ballance, 1992).

Esta heterogeneidad del hábitat, asociada a los requerimientos biológicos de cada especie, promueve la generación de nichos ecológicos especializados, lo que propicia la diferenciación de las poblaciones de muchas especies marinas (Natoli, Birkun, Aguilar, López y Hoelzel, 2005). Las toninas (Tursiops truncatus) establecen asociaciones de tipo fisión-fusión, es decir, varían su composición grupal por hora y por día, lo que les permite tener un tipo de estructura social que les proporciona capacidades importantes de aprendizaje, de alimentación, reproducción, defensa y comunicación (Connor, Well, Mann y Read, 2000). Este tipo de asociaciones también les permite explotar una amplia variedad de hábitats tanto oceánicos como costeros de casi todos los océanos del mundo (Díaz-Gamboa, 2004; Wells y Scott, 2002).

En el golfo de California se han realizado estudios de genética poblacional (Segura, 2004, 2011) y de isótopos estables (Díaz-Gamboa, 2004) que han permitido diferenciar a 2 ecotipos de toninas: un ecotipo costero y otro pelágico (Segura, 2011; Vázquez-Castán, Serrano y Galindo, 2009). Estos ecotipos van a presentar diferencias en su morfología, pigmentación, alimentación, distribución y tipo de asociación grupal (Díaz-Gamboa, 2004; Segura, 2004, 2011). Además, existen factores biológicos y oceanográficos que favorecen la distribución y el comportamiento de cada uno de los ecotipos de toninas, tales como la distribución de sus presas y los relieves marinos (GuevaraAguirre, 2011; Jaquet y Gendron, 2002), los que generan una dependencia local de diversas condiciones ecológicas; de este modo se apoya la hipótesis de la dependencia del «hábitat local».

La mayoría de estudios se han centrado en el ecotipo costero, y se ha definido que su distribución se da en aguas someras de entre 0.5 y $20 \mathrm{~m}$ de profundidad con baja visibilidad y de fondos arenosos, principalmente asociados con áreas de una alta productividad primaria como las desembocaduras de ríos y lagunas costeras (Cubero, 2007; Gallo-Reynoso, Égido-Villareal, Coria-Galindo y Saldaña, 2006; GuevaraAguirre, 2011; Guevara-Aguirre y Gallo-Reynoso, 2015; Marcín-Medina, 2010; Patiño-Valencia, Vargas y Díaz, 2008; Reza-García, 2001; Segura, 2004). El ecotipo costero muestra patrones de movimiento limitados (Connor et al., 2000; Cubero, 2007), mientras que el ecotipo oceánico presenta movimientos menos restringidos y se encuentra asociado a zonas de alta productividad; se ha observado que algunas poblaciones del ecotipo oceánico pueden ser residentes en los alrededores de islas oceánicas (Klatsky, Wells y Sweeney, 2007).

Las diferencias de alimentación entre las toninas costeras y oceánicas se deben a que sus presas provienen de diferentes ecosistemas (Díaz-Gamboa, 2004). El ecotipo costero aprovecha las poblaciones de presas locales (Díaz-Gamboa, 2004), mientras que las toninas oceánicas se alimentan principalmente de peces epipelágicos y cefalópodos (Barros, Parsons y Jefferson, 2000). Por esta razón, en el golfo de California el ecotipo oceánico presenta una posición trófica similar a los grupos de hembras y jóvenes de cachalotes (Physeter macrocephalus) y de las ballenas piloto (Globicephala macrorhynchus) con los cuales se llega a asociar (Díaz-Gamboa, 2004; Gallo-Reynoso, 1984). Tomando en cuenta lo anterior, el objetivo de este trabajo fue definir las características fisiográficas y oceanográficas de la cuenca de Guaymas en el golfo de California y establecer la relación de estas características con el uso del hábitat de los 2 ecotipos de toninas para generar modelos de nicho ecológico y establecer si existen variables que determinen el hábitat que utilizan estos organismos.

\section{Materiales y métodos}

El muestreo se realizó en el golfo de California, en 2 áreas de la cuenca de Guaymas. Los muestreos para el ecotipo costero se llevaron a cabo en los sistemas lagunares de la bahía de las Guásimas. Los sistemas tienen su origen en la llanura deltaica del río Yaqui $\left(27^{\circ} 50^{\prime} \mathrm{N}-110^{\circ} 35^{\prime} \mathrm{O}\right)$ (fig. 1), constituyendo una zona somera con gradientes hipersalinos; en época de lluvias extremas los gradientes pueden ser hiposalinos. La batimetría y la fisiografía son variables. Geomorfológicamente, corresponde a un valle inundado y ocupa planicies costeras anchas y de poco relieve (Gallo-Reynoso et al., 2006).

Los muestreos del ecotipo oceánico se llevaron a cabo en la porción noroeste de la cuenca de Guaymas, en la costa central de Sonora, en el área oceánica que se extiende desde la isla San Pedro Nolasco hasta la isla San Esteban. Las corrientes superficiales están influenciadas por un patrón estacional de vientos y de mareas semidiurnas. Durante el invierno los vientos provienen del noroeste, lo que produce surgencias fuertes en la zona costera continental, mientras que durante el verano los vientos soplan del sureste y producen surgencias débiles en la región costera de la península de Baja California (Roden, 1964; Santamaría del Ángel y Álvarez-Borrego, 1994). La batimetría de la zona corresponde a una gran porción de la plataforma costera, del talud continental y de los fondos asociados a las islas San Pedro Nolasco, San Pedro Mártir y San Esteban. En la isla San Pedro Nolasco se encuentra un bajo profundo (de 98 a 300 m) que actúa como dique de contención de las surgencias estacionales en el área (Pérez y Sosa, 2014).

\section{Análisis de datos}

Para determinar el uso de hábitat del ecotipo costero se realizaron 13 muestreos para recorrer la zona costera de las Guásimas entre el otoño de 2012 y el verano de 2013; cada muestreo equivale a un recorrido completo a la laguna de las Guásimas y a sus sistemas lagunares adyacentes. Específicamente, se realizaron 3 muestreos en el otoño de 2012, 4 en el invierno de 2013, 3 en la primavera de 2013 y 3 en el verano de 2013. Se trabajó un total de 64 h, de las cuales 24 h se destinaron a la observación directa 


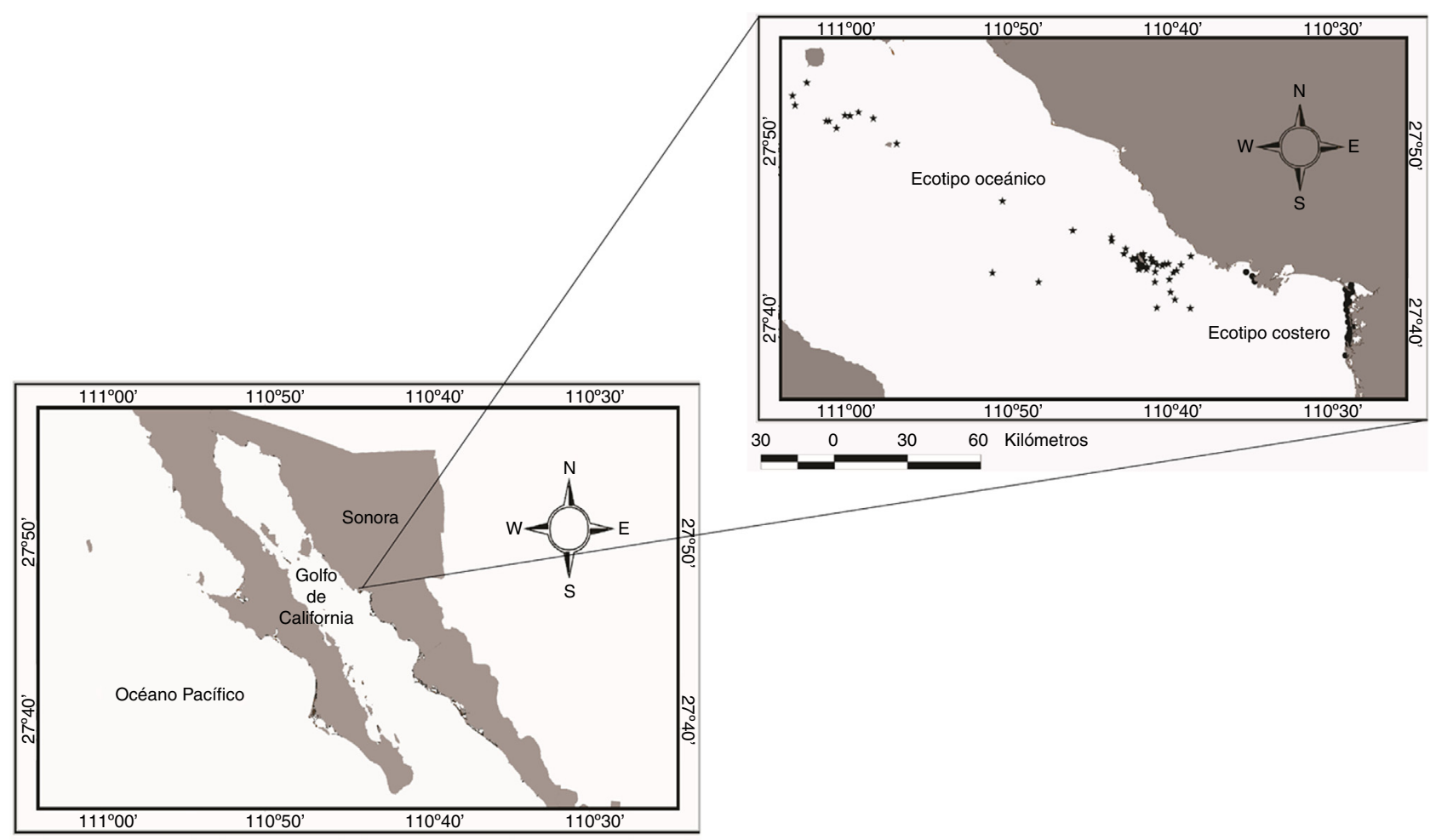

Figura 1. Área de estudio de las toninas costeras y oceánicas en la cuenca de Guaymas, golfo de California, México. Las estrellas representan los avistamientos del ecotipo oceánico; los círculos representan los avistamientos del ecotipo costero.

de las manadas de toninas. Los datos detallados del esfuerzo realizado y la cantidad de toninas que se observaron por estación climática se encuentran en Guevara-Aguirre y Gallo-Reynoso (2015).

Se realizaron 16 muestreos para caracterizar el área oceánica en la cuenca de Guaymas; cada muestreo equivale a un recorrido a la zona oceánica de la cuenca de Guaymas, principalmente en los alrededores de la isla San Pedro Nolasco y la isla San Pedro Mártir. El muestreo comenzó en la primavera de 2013 hasta el invierno de 2014; se realizaron 4 muestreos en la primavera de 2013, 5 en el verano de 2013, 4 en el otoño de 2013 y 3 en el invierno de 2014. Se trabajó un total de $109.3 \mathrm{~h}$, de las cuales $14 \mathrm{~h}$ se destinaron a la observación directa de las manadas de toninas oceánicas (Guevara-Aguirre y Gallo-Reynoso, 2015). Para la modelación general de presencia/ausencia (nicho ecológico) de los ecotipos costero y oceánico se realizaron 5 salidas más a la zona oceánica ( 3 en el otoño de 2012 y 2 en el invierno de 2013), por lo que en total se realizaron 21 salidas al área oceánica.

Los recorridos se realizaron en pangas de fibra de vidrio de $7 \mathrm{~m}$ de eslora con motor fuera de borda de 60-75 HP. En 4 salidas de campo (mayo, julio, septiembre y octubre) el recorrido se llevó a cabo en una embarcación tipo yate de $15 \mathrm{~m}$ de eslora. Las embarcaciones se guiaron mediante un geoposicionador y se controló la velocidad de desplazamiento. El objetivo primordial de las navegaciones fue la búsqueda específica de las manadas de toninas, para obtener la localización geográfica precisa y caracterizar sus hábitats (así, la batimetría especifica del avistamiento). De 2 a 3 personas equipadas con binoculares con potencia de $10 \times 50$ realizaron las observaciones a una altura de 2 a $4 \mathrm{~m}$.
Una vez localizadas las toninas, la embarcación se aproximaba y se determinaba el número de grupos focales, el cual se define como un grupo de toninas en aparente asociación que se mueve en una misma dirección y realiza la misma actividad (Guevara-Aguirre y Gallo-Reynoso, 2015). Los comportamientos se clasificaron en 4 categorías, las cuales se han utilizado en estudios anteriores, ya que se ha observado que determinan la totalidad de comportamientos que presentan las toninas: alimentación, descanso, tránsito y social (Ballance, 1992). Una vez definido el comportamiento de las toninas, la embarcación se detenía y se medían 6 parámetros in situ con equipos especializados para cada variable: la profundidad se midió con una ecosonda (Furuno-GPS $1850 \mathrm{WF}$ dual de $50-200 \mathrm{kHz}$ con profundidad máxima de $2,000 \mathrm{~m}$ ) para la zona oceánica y una ecosonda manual (Humminbird Wide-Vision, Techsonic Industries Inc., Eufaula, Alabama, con profundidad máxima de 200 m) para la zona costera; el color del mar se midió con un colorímetro LaMotte (5907), que utiliza la escala de Forel-Ule; la transparencia del agua se midió con un disco de Secchi; el estado del mar se estimó con la escala de Beaufort; la salinidad se midió con un refractómetro marca Vista A366ATC (0-10\%o Sal.), y la temperatura superficial se midió con un multiparámetro marca HANNA modelo HI9828. Todos los instrumentos fueron calibrados antes de cada salida.

Otras 4 variables oceanográficas se obtuvieron en el laboratorio: la concentración de clorofila se obtuvo de la National Aeronautics and Space Administration (NASA), la distancia a la costa con el programa Arc View GIS 3.2, la pendiente del terreno se determinó al realizar un levantamiento batimétrico usando la base de datos de ETOPO1 Global Relief Model proporcionado 
por la NASA, y la batimetría con la ecosonda (Furuno-GPS) antes mencionada, que se utilizó para realizar el levantamiento batimétrico de la cuenca de Guaymas (Pérez y Sosa, 2014), y mediante el uso de ArcView GIS 3.2 se generaron los mapas batimétricos y se obtuvo la pendiente del fondo de las zonas de estudio. La densidad del agua se obtuvo con un convertidor en línea, que utiliza la temperatura y la salinidad del agua y genera valores en kilogramos por metro cúbico $\left(\mathrm{kg} / \mathrm{m}^{3}\right)$.

Estas 10 variables ambientales se seleccionaron para ser utilizadas porque en estudios previos realizados en el golfo de California se observó su importancia para determinar la distribución de los peces que son presas potenciales de las toninas y que, por tanto, determinan de forma indirecta la distribución de estos mamíferos marinos (Guevara-Aguirre, 2011; MarcínMedina, 2010; Martínez-Serrano, Serrano, Heckel y Schramm, 2011; Patiño-Valencia et al., 2008).

Para determinar el uso de hábitat de cada uno de los ecotipos (costero y oceánico) por separado se analizaron los comportamientos de cada ecotipo de toninas de acuerdo a diferentes categorías de tiempo. Estas categorías de tiempo fueron establecidas con una separación de $3 \mathrm{~h}$, desde el inicio de los muestreos hasta la finalización de los mismos, con la finalidad de determinar si la hora del día tiene una influencia en los patrones de comportamiento de las toninas. Para cada ecotipo se utilizaron diferentes categorías de tiempo, debido a que los muestreos de ambos ecotipos no tuvieron la misma duración, ni pudieron comenzar a la misma hora por cuestiones de logística, siendo de mayor duración los muestreos del ecotipo oceánico, por lo que el análisis que se realizó para definir la preferencia de los comportamientos por alguna categoría de tiempo es independiente para cada ecotipo.

Para el ecotipo costero se utilizaron 3 categorías de tiempo para cubrir la totalidad de los muestreos, ya que los muestreos se llevaron a cabo de las 6:00 am hasta las $15: 00 \mathrm{pm}$, siendo estos: H1 6:00-8:59 h, H2 9:00-11:59 h y H3 12:00-15:00 h. Posteriormente se realizó un cuadro con el número de ocasiones que las toninas realizaban algún comportamiento en cada una de las categorías de tiempo. Para el ecotipo oceánico se establecieron 5 categorías de tiempo debido a que los muestreos en la zona oceánica fueron de las 6:00 am hasta que la luz del sol permitía avistar toninas: H1 6:00-8:59h, H2 9:00-11:59h, H3 12:00-14:59 h, H4 15:00-17:59 h y H5 18:00-21:00 h, y se realizó el mismo conteo de aparición de comportamientos por hora.

Se hizo un análisis exploratorio de correspondencia para cada ecotipo de tonina por separado para describir la relación entre los comportamientos observados de cada ecotipo y la hora del día, a fin de determinar los patrones de comportamiento de cada uno de los 2 ecotipos de toninas. Posteriormente se hizo un modelo lineal generalizado (GLM) para cada ecotipo para determinar si alguna de las 10 variables oceanográficas medidas tiene una correlación con alguno de los 4 comportamientos que se observaron en los 2 ecotipos de toninas; los modelos que se hicieron fueron de presencia/ausencia, por lo que los valores de presencia fueron los obtenidos del comportamiento que se iba a modelar, mientras que los datos de ausencia fueron los datos de los otros 3 comportamientos, por lo que en total se realizaron 4 GLM por ecotipo. Con la finalidad de conocer qué modelo elegir se utilizó el criterio de Información Akaike (Akaike Information Criterion [AIC]) para comparar modelos similares con distintos grados de complejidad o modelos iguales (mismas variables) pero con funciones de vínculo distintas. El modelo que presentó un mejor ajuste - y por lo tanto el que se eligió- fue el que tuvo el valor de AIC más pequeño. Posteriormente se realizó otro GLM con las variables del modelo anteriormente elegido y se calculó la probabilidad de presencia de los comportamientos de las toninas. Todos los análisis estadísticos se realizaron utilizando el programa estadístico Statistica 7.

\section{Nicho ecológico}

Se realizó un GLM para cada ecotipo utilizando datos de presencia y ausencia; los datos de ausencia fueron tomados de estaciones predeterminadas y de datos de avistamientos de otras especies de mamíferos marinos que se observaron en cada una de las zonas de estudio durante los años de muestreo. El AIC fue nuevamente usado para comparar modelos similares con diferentes grados de complejidad o modelos similares pero con diferente función de vínculo, el modelo elegido fue el que menor valor de AIC presentó, lo que indica un mejor ajuste. El objetivo del modelado del nicho ecológico es el de identificar los sitios idóneos, en el área de estudio, para la supervivencia de los 2 ecotipos de toninas por medio de la identificación de sus requerimientos ambientales, que conforman su nicho fundamental (Soberón y Nakamura, 2009).

\section{Resultados}

Durante los muestreos al área costera se realizó un esfuerzo de búsqueda efectiva de $64 \mathrm{~h}$, en las cuales se observaron 74 manadas con 452 toninas. Durante los muestreos al área oceánica se registraron 95.4 horas efectivas de búsqueda de toninas, en donde se observaron 2,183 toninas agrupadas en 45 manadas; en 2 de los muestreos (abril 2013 y marzo 2014) no se observaron toninas oceánicas en la zona de estudio (GuevaraAguirre y Gallo-Reynoso, 2015). Para la modelación general (nicho ecológico) se incrementó el número de muestreos que se realizaron, por lo que se registraron 2,295 individuos agrupados en 55 manadas de toninas oceánicas.

\section{Comportamiento por bloques de tiempo}

El análisis exploratorio de correspondencia para las toninas costeras (fig. 2) indica que los comportamientos de «alimentación» y «tránsito» se relacionan con $\mathrm{H} 1$ y H2. El «descanso» está más relacionado con $\mathrm{H} 3$ que con $\mathrm{H} 2$, mientras que el comportamiento «social» se relaciona con $\mathrm{H} 2$. El análisis de correspondencia del ecotipo oceánico nos indica que el comportamiento de «alimentación» se relaciona con H1 y $\mathrm{H} 5$, el comportamiento «descanso» se relaciona con $\mathrm{H} 4$, el comportamiento «tránsito» se relaciona con $\mathrm{H} 2$ y $\mathrm{H} 4$, y el comportamiento «social» se relaciona con $\mathrm{H} 3$. 

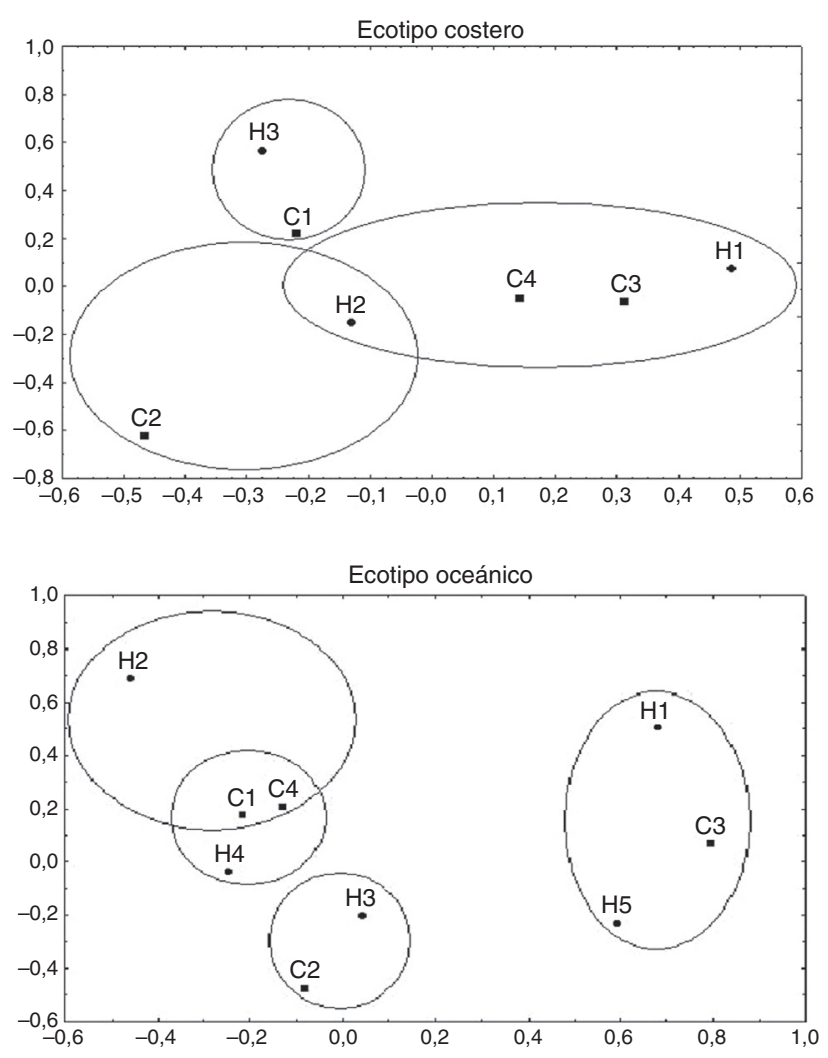

Figura 2. Análisis de correspondencia de los comportamientos y las horas del día de las toninas costeras y oceánicas $(\mathrm{C} 1$ : descanso; $\mathrm{C} 2$ : social; C3: alimentación; C4: tránsito).

\section{Uso de hábitat}

Toninas costeras. El GLM de los comportamientos del ecotipo costero utilizando las 10 variables ambientales (tabla 1) indica que la temperatura superficial está relacionada negativamente con los comportamientos descanso y social; así, menores temperaturas (entre 16 y $20^{\circ} \mathrm{C}$ ) incrementan la probabilidad de observar al ecotipo costero en descanso y socializando. La visibilidad y la salinidad determinan la aparición del comportamiento alimentación, ambas variables con una relación negativa: así, a menor visibilidad $(<2.5 \mathrm{~m})$ y menor salinidad $(27.5$ y $30 \%$ ) se incrementa la probabilidad de observar el comportamiento alimentación. Finalmente, 4 variables ambientales determinan el comportamiento de tránsito (salinidad, color de mar, distancia a la costa y la pendiente del fondo), todas con una relación positiva, por lo que los lugares que presenten características de salinidad $>37 \%$, color del mar $>15$ en la escala Forel-Ule, distancia a la costa $>$ a $1 \mathrm{~km}$ y pendientes $>0.4^{\circ}$, van a incrementar la probabilidad de observar el comportamiento de tránsito en el ecotipo costero.

Toninas oceánicas. El GLM de los comportamientos del ecotipo oceánico (tabla 2) indica una relación negativa entre el comportamiento de descanso con las variables profundidad y color del mar; en otras palabras, el ecotipo oceánico descansa en lugares con baja profundidad (100-200 m) y en aguas claras (con un valor de 1 en la escala de Forel-Ule). El comportamiento social tiene una relación negativa con el estado del mar, por lo que la probabilidad de observar este comportamiento se incrementa en lugares con el mar en calma. El comportamiento alimentación tiene una relación positiva con la profundidad, lo que significa que el ecotipo oceánico se alimenta en lugares más profundos (entre 400 y $800 \mathrm{~m}$ ). Finalmente, el GLM indica una relación positiva entre el comportamiento tránsito y la temperatura superficial, el color de mar y la pendiente del fondo, sugiriendo que el ecotipo oceánico transita en lugares con altas temperaturas $\left(>25^{\circ} \mathrm{C}\right)$, pendientes pronunciadas $\left(>30^{\circ}\right)$ y aguas verdosas con un valor mayor de 6 en la escala de Forel-Ule.

\section{Nicho ecológico}

Modelo lineal generalizado (presencia/ausencia). El modelo para el ecotipo costero demuestra que 3 variables ambientales

Tabla 1

Modelo lineal generalizado para los comportamientos de las manadas de toninas costeras de acuerdo a las 10 variables medidas. Se muestra el valor Akaike (AIC) y las relaciones con las diferentes variables.

\begin{tabular}{lllllll}
\hline \multicolumn{7}{c}{ Ecotipo costero } \\
\hline & AIC & Temperatura superficial & Visibilidad & Salinidad & Color del mar & Distancia a la costa \\
\hline Descanso & 100.780 & -0.083 & & & & \\
Social & 48.059 & -0.119 & -0.418 & -0.300 & & 0.001 \\
Alimentación & 94.090 & & & 0.078 & 0.166 & 4.929 \\
Tránsito & 55.157 & & & & \\
\hline
\end{tabular}

Tabla 2

Modelo lineal generalizado para los comportamientos de las manadas de toninas oceánicas de acuerdo a las 10 variables medidas. Se muestra el valor Akaike (AIC) y las relaciones con las diferentes variables.

\begin{tabular}{llcccc}
\hline & & & & \\
\hline & AIC & Temperatura superficial & Profundidad & Color del mar & Estado del mar \\
\hline Descanso & 53.488 & & -0.004 & -0.150 & -0.853 \\
Social & 62.636 & & & \\
Alimentación & 52.222 & 0.067 & 0.05 & 0.006 & 0.093 \\
Tránsito & 58.279 & 0.067 & & \\
\hline
\end{tabular}


Tabla 3

Variables importantes que determinan la presencia de cada ecotipo de tonina indicada por el modelo lineal generalizado.

\begin{tabular}{llccc}
\hline & & GLM presencia/ausencia & \\
\hline Ecotipo & AIC & Pendiente & Concentración de clorofila & Distancia a la costa \\
\hline Costero & 54.702 & -0.714 & 1.795 \\
Oceánico & 94.672 & -0.407 & 1.742 & -0.232 \\
\hline
\end{tabular}

(distancia a la costa, concentración de clorofila y color del mar) determinan la presencia de este ecotipo. Para ello, este ecotipo se encuentra distribuido a una mayor distancia de la costa, preferentemente a una distancia de $(\overline{\mathrm{X}}=0.9 \pm 0.6 \mathrm{~km})$ sobre la plataforma continental $(<5 \mathrm{~km}$ de la costa), con bajas concentraciones de clorofila $\left(0-2 \mathrm{mg} / \mathrm{m}^{3}\right)$ y en lugares con aguas con un valor mayor de 15 en la escala de Forel-Ule (muy cafés). El modelo para el ecotipo oceánico indica que la pendiente, la concentración de clorofila y la distancia a la costa determinan la presencia de este ecotipo, lo que significa que serán encontrados en lugares con baja pendiente $\left(0-5^{\circ}\right)$, con una alta concentración de clorofila $\left(>2 \mathrm{mg} / \mathrm{m}^{3}\right)$ y cercanos a la costa, pero siempre después del talud continental ( $>5 \mathrm{~km})$ (tabla 3$)$.

\section{Discusión}

El golfo de California es uno de los ecosistemas más biodiversos, dinámicos y productivos del planeta, debido a que presenta 3 mecanismos principales de fertilización: las surgencias inducidas por la batimetría y por el viento, la mezcla de agua producida por los cambios semidiurnos de las mareas y la circulación termohalina, lo que lo convierte en una región con gran complejidad ecológica y de una elevada productividad primaria (ÁlvarezBorrego, 2002). Esta productividad permite la presencia de una gran cantidad de mamíferos marinos, como es el caso de las toninas costeras, que aprovechan los lugares de alta actividad biológica como las descargas de ríos, zonas estuarinas y áreas de surgencia (Baumgartner, Mullin, May y Leming, 2001), y del mismo modo que el ecotipo oceánico, se distribuyen en lugares de alta productividad primaria y de relieves marinos complejos (Guevara-Aguirre, 2011; Jaquet y Gendron, 2002; Pérez y Sosa, 2014).

\section{Uso de hábitat}

Toninas costeras. Los patrones de actividad de las toninas se modifican de manera estacional y espacial. De acuerdo al hábitat que ocupen existen variables ecológicas y ambientales que afectan su distribución y abundancia, tales como: la distribución de sus presas, la hora del día, la profundidad del agua, la topografía del fondo, el flujo de la marea y algunas actividades humanas (Cubero, 2007). Este cambio en las variables puede fragmentar el hábitat en varios microhábitats, propiciando una distribución de las toninas en función del uso que hagan de los mismos. Estos microhábitats a su vez generan presión selectiva sobre algunos individuos de una población, produciendo que una misma especie presente diferentes tipos, los cuales están adaptados a un conjunto de características del hábitat; estos grupos son llamados ecotipos. Por ejemplo, algunos microhábitats les pueden otorgar protección contra los depredadores, mientras que otros les pueden proporcionar una mayor concentración de alimento (Eierman y Connor, 2014).

El ecotipo costero estuvo presente en toda la zona de estudio, y los lugares con mayor densidad fueron la boca de la bahía de las Guásimas y la boca del estero Los Algodones (GuevaraAguirre y Gallo-Reynoso, 2015). En esta zona la temperatura superficial del agua determinó los comportamientos de descanso y social. Marcín-Medina (2010) anteriormente había observado este patrón en la bahía de La Paz, y menciona que a una temperatura de $25-27^{\circ} \mathrm{C}$ existe una mayor posibilidad de encontrar a las toninas en descanso, posiblemente debido a que las toninas costeras pueden descansar y socializar después de alimentarse. Estas toninas en descanso se distribuyeron principalmente en las bocas de los sistemas estuarinos de la bahía de las Guásimas, ya que estas zonas les proporcionan protección, lo que les permite descansar y cuidar a las crías de posibles depredadores (Gallo-Reynoso et al., 2006).

El hecho de que las variables que determinan el comportamiento de alimentación en las toninas costeras sean las bajas visibilidad y salinidad es debido a que el ecotipo costero aprovecha estas condiciones hidrológicas que se encuentran principalmente en la boca de los estuarios (Cubero, 2007; Guevara-Aguirre y Gallo-Reynoso, 2015), ya que las toninas utilizan estas áreas como zonas de alimentación, debido a la abundancia y a la densidad de peces que las bocas de los estuarios presentan; las toninas han desarrollado una estrategia de alimentación en la que se mueven en contra de la corriente de marea permaneciendo en el borde entre aguas con diferente visibilidad para facilitar la captura de sus presas, y de esta forma maximizan la detección y la captura (Guevara-Aguirre, 2011; Marcín-Medina, 2010). Este comportamiento se presenta principalmente en lugares con entradas de agua continental, como los ríos, que proveen agua dulce y turbulencia afectando la visibilidad con los sedimentos que acarrean, lo que explica que la distribución de las toninas en alimentación se encuentre principalmente en la boca del estero Los Algodones (en donde hay un ramal del delta del río Yaqui).

Investigaciones anteriores han sugerido que las zonas con mantos de algas, zonas rocosas y aguas muy turbias pueden proveer refugio a los peces, por lo que la preferencia de las toninas por zonas arenosas se deba a que sus presas no pueden esconderse en este tipo de sedimento (Morteo, Heckel, Defran y Schramm, 2004). Sin embargo, las zonas rocosas y turbias no son un inconveniente para la alimentación de las toninas, ya que utilizan su sistema acústico de manera activa para detectar a sus presas y su entorno, generando una gran cantidad de trenes 
de ecolocalización, como se presentó en nuestra zona de estudio (Bazúa-Durán y Herrera-Hernández, 2007). La hora del día también es un factor que influye en este comportamiento, ya que es durante la mañana cuando se ha registrado este comportamiento.

El comportamiento de tránsito básicamente aparece cuando las toninas van a buscar lugares propicios de alimentación y descanso, por lo que este comportamiento se presenta en lugares alejados a la línea de costa $(>1 \mathrm{~km})$, pero siempre sobre la plataforma continental, en donde la pendiente y la salinidad se incrementan debido a la presencia del talud continental y a la baja influencia de las entradas de agua dulce. Esto concuerda con lo reportado por Ballance (1992) en la bahía de Kino, quien menciona que el ecotipo costero viaja alejado de la línea de costa $(>5 \mathrm{~km})$, por lo que es posible que estas características no favorezcan la presencia de las toninas y por lo tanto viajen para conseguir alimento o lugares de descanso.

Toninas oceánicas. El ecotipo oceánico estuvo distribuido en los alrededores de las islas San Pedro Nolasco y San Pedro Mártir en la cuenca de Guaymas (Guevara-Aguirre y Gallo-Reynoso, 2015). En esta zona las variables ambientales y la hora del día determinaron la presencia de los diferentes comportamientos. El ecotipo oceánico se alimenta muy temprano y por la noche. Este comportamiento se observó con el incremento de la profundidad, probablemente por la migración vertical diaria que realizan los eufáusidos y mictófidos, ya que se ha observado que durante las primeras horas del día y en la noche algunas especies de eufáusidos y mictófidos se distribuyen superficialmente para alimentarse de fitoplancton y zooplancton (Tremblay, 2008), reduciendo la mortalidad ocasionada por depredadores epipelágicos y evitando los rayos ultravioleta del sol. Durante el resto del día, los eufáusidos y los mictófidos migran a aguas más profundas, por lo que esta migración genera una distribución de las posibles presas que son aprovechadas por las toninas.

Este comportamiento de alimentación aparece en lugares con mayor profundidad (entre 400 y $800 \mathrm{~m}$ ), ya que se ha reportado que el ecotipo oceánico presenta un nivel trófico similar al de los juveniles de los cachalotes (Physeter macrocephalus) (Díaz-Gamboa, 2004), con los cuales se llega a asociar, al igual que con las ballenas piloto (Globicephala macrorhynchus) (Gallo-Reynoso, Égido-Villareal y Coria-Galindo, 2009), ya que se alimentan principalmente de peces pelágicos y de calamar gigante (Dosidicus gigas), los cuales presentan una mayor abundancia en la isobata de los $600 \mathrm{~m}$ (Gallo-Reynoso et al., 2009; Gendron, 2000).

El análisis de correspondencia nos indica que el comportamiento de tránsito aparece después del comportamiento de alimentación, en lugares con alta temperatura superficial $\left(>25^{\circ} \mathrm{C}\right)$, en aguas verdosas y con pendientes pronunciadas $\left(>30^{\circ}\right)$, posiblemente porque estas características no favorecen la distribución de posibles presas de las toninas. El máximo número de toninas observadas realizando este comportamiento de tránsito fue durante el verano, cuando existe una incursión de agua caliente y oligotrófica del Pacífico en el golfo de California (Guevara-Aguirre y Gallo-Reynoso, 2015), lo que reduce la productividad primaria (Álvarez-Borrego, 2002; Pérez-Arvizu, Aragón-Noriega y Espinosa-Carreón, 2013), por lo que es posible que estos lugares puedan ser usados como rutas de búsqueda de alimento o de descanso.

El comportamiento social en las toninas oceánicas se presenta principalmente de las 12:00 a las 14:59h en lugares con poco oleaje, lo que posiblemente se debe a que en este horario las toninas ya se han alimentado y pueden utilizar el tiempo para esparcimiento, además de que las condiciones de poco oleaje del mar son idóneas para socializar. La mayoría de los individuos que se encontraron realizando este comportamiento fueron individuos juveniles, ya que se sabe que estos son más curiosos $\mathrm{y}$, como que ya no necesitan el resguardo de la madre, pueden realizar esta actividad de manera habitual.

Finalmente, el ecotipo oceánico descansa durante la tarde (entre las 15:00 y 17:59h) en lugares con menor profundidad (100-200 m) y aguas claras (azules), por lo que es posible que no exista mucho alimento para las toninas (Santamaría del Ángel y Álvarez-Borrego, 1994). También existe la posibilidad de que los lugares con menor profundidad y buena visibilidad puedan proporcionar a las toninas una zona de protección contra depredadores, debido a que la luz puede penetrar en estas profundidades y es más fácil ver a un potencial depredador.

\section{Nicho ecológico}

La heterogeneidad del hábitat y los requerimientos biológicos de las especies pueden promover la generación de nichos ecológicos especializados y tener la capacidad de generar patrones de distribución y del uso del hábitat, que propicia la diferenciación y la especiación de muchas poblaciones de especies, incluyendo a los mamíferos marinos (Hoelzel, 1998; SuárezEsteban y Miján, 2011). Estos casos de polimorfismo apoyan la hipótesis del hábitat local, el cual propicia la diferenciación de la población en ausencia de barreras físicas, debido a que las especializaciones intraespecíficas reflejan un uso diferencial del nicho.

En general los cetáceos presentan una compleja variación espaciotemporal en su distribución, asociada principalmente a las condiciones oceanográficas, la geomorfología del suelo marino (geología estructural, topografía y tipo de sedimento) y a las condiciones hidrodinámicas que otorgan características específicas en su hábitat para la reproducción, alimentación y crianza (Medrano y Urbán, 2002). En el caso particular de las toninas se ha reportado que pueden ocupar nichos costeros y oceánicos (Wells y Scott, 2002), y que las variables ambientales más importantes que van a determinar su presencia son la temperatura, la salinidad, la profundidad, el tipo de sedimento y la distancia a la costa (Cubero, 2007; Guevara-Aguirre, 2011; Morteo et al., 2004). Sin embargo, en este estudio las variables que determinaron y diferenciaron el hábitat de los 2 ecotipos de toninas fueron la pendiente del fondo, la concentración de clorofila, la distancia a la costa y el color del mar. La concentración de clorofila y la distancia a la costa influyeron en la presencia de ambos ecotipos, mientras que la pendiente influyó solamente en la presencia del ecotipo oceánico, y el color del mar solamente influyó en la presencia del ecotipo costero.

La distancia a la costa es una variable que se utilizó para determinar si la distribución de los 2 ecotipos se daba sobre 
la plataforma o a partir del talud continental de acuerdo a la batimetría de la zona. La mayor probabilidad de encontrar a las toninas costeras es lejos de la costa, dentro del rango de $5 \mathrm{~km}$ de la costa, preferentemente a una distancia promedio de $\overline{\mathrm{X}}$ $=0.9 \pm 0.6 \mathrm{~km}$. Es importante mencionar que la mayor distancia a la costa encontrada para las toninas costeras fue de $2.5 \mathrm{~km}$, similar a lo reportado por Martínez-Serrano et al. (2011), quienes indican que en el golfo de México las manadas de toninas se encontraron a una distancia promedio de la costa de $2.5 \mathrm{~km}$. La plataforma continental en el golfo de México es más extensa - de 37 a $80 \mathrm{~km}$ de sur a norte (De la Lanza-Espino, 1991) — que en la región central del golfo de California, en donde la plataforma continental es de apenas $5 \mathrm{~km}$ (Lugo, 1986), por lo que la distribución de las toninas costeras se dará por sobre la plataforma continental y en lugares alejados de la costa, como ya se había reportado en el golfo de California y en otras partes de México (Ballance, 1992; Cubero, 2007; Guevara-Aguirre, 2011).

En el caso de las toninas oceánicas la distancia a la costa mostró una relación negativa, y es importante mencionar que la distancia promedio en las que se encontraron a las toninas oceánicas fue de $23.79 \mathrm{~km}$. En la zona central de las costas de Sonora la plataforma continental es muy estrecha, aproximadamente de $5 \mathrm{~km}$. La mayor densidad de avistamientos se dio en los alrededores de la isla San Pedro Nolasco, que se encuentran aproximadamente a $15 \mathrm{~km}$ de la línea de costa; entre la isla y la costa se encuentra un canal de aproximadamente $300 \mathrm{~m}$ de profundidad (Guevara-Aguirre y Gallo-Reynoso, 2015). Esta distribución concuerda con lo encontrado por Segura (2004), quien reporta que la distribución del ecotipo oceánico se da en zonas principalmente con profundidades superiores a los $300 \mathrm{~m}$, por fuera de la plataforma continental.

Como se ha mencionado con anterioridad, la distribución de los mamíferos marinos está determinada principalmente por los movimientos de sus presas y por la concentración de clorofila en la zona (productividad primaria) (Jaquet y Gendron, 2002), factores que diferencian a los 2 ecotipos de toninas. La relación negativa de esta variable con el ecotipo costero no concuerda con lo reportado por Medrano y Urbán (2002), quienes indican que las toninas y los mamíferos marinos en general están relacionados con lugares de alta productividad primaria, como las bocas de los estuarios y lagunas costeras, dado que estos lugares cuentan con un mayor número de presas que son aprovechadas por los mamíferos marinos. Sin embargo, encontramos que el color del mar fue una variable importante en la presencia del ecotipo costero debido a que la distribución de las manadas se presentó en las bocas de los ríos, caracterizada por presentar una gran turbidez.

Estos lugares tienen un gran aporte de terrígenos y una alta productividad primaria, siendo el principal factor ambiental que afecta a las zonas costeras, ya que los ríos son los «responsables» del ingreso a las áreas costeras de cargas importantes de nutrientes, así como de componentes orgánicos e inorgánicos. El color de mar, según la escala Forel-Ule, describe la transparencia del agua y por lo tanto también ayuda a clasificar la actividad biológica bruta. En el caso de las toninas costeras de las Guásimas, los valores que dominaron son los pertenecientes a la escala Ule, que están destinados a aguas turbias con gran cantidad de diatomeas, por lo que de manera indirecta el color del mar indica que el ecotipo costero se presenta en lugares muy productivos, al menos localmente (Aguirre, 2002).

La relación positiva encontrada entre la concentración de clorofila y la presencia del ecotipo oceánico concuerda con lo reportado en el golfo de California (Pérez y Sosa, 2014). Esta relación se ha observado en muchas especies de mamíferos marinos que lo habitan, debido a que su alta productividad genera una gran complejidad ecológica, creando ambientes idóneos para muchas especies de mamíferos marinos, en particular de especies que habitan ambientes más oceánicos que costeros como lo pueden ser el rorcual común (Balaenoptera physalus), la ballena azul (Balaenoptera musculus), las ballenas jorobadas (Megaptera novaeangliae) y el rorcual tropical (Balaenoptera edeni). Estas especies aprovechan el alimento que se distribuye en la columna de agua ocasionado por las surgencias del golfo de California (Medrano y Urbán, 2002).

Esta relación también se ha observado en odontocetos como el delfín común de rostro largo (Delphinus capensis), que al igual que las ballenas piloto (Globicephala macrorhynchus) y los zifios de Cuvier (Ziphius cavirostris) y de Baird (Berardius bairdii), se distribuyen sobre los límites entre la plataforma continental y el talud continental con un gran relieve topográfico que genera surgencias físicas, moviéndose de acuerdo con la distribución de su alimento, calamares, rayas y pulpos (Guerrero-Ruiz, Urbán-Ramírez y Rojas-Bracho, 2006).

La batimetría es un factor importante en la presencia de muchos odontocetos que habitan el golfo de California, ya que se distribuyen en aguas oceánicas con una elevada pendiente y una complejidad topográfica (Guerrero-Ruiz et al., 2006), en particular en la parte sur de la isla San Pedro Nolasco, en donde se tiene la presencia de un bajo profundo que produce una surgencia física, llenando la columna de agua de nutrientes. En referencia con esto, la pendiente fue una variable importante en la presencia de las toninas, ya que se ha reportado que las toninas prefieren zonas escarpadas en donde la geomorfología favorece la presencia de surgencias (Pérez y Sosa, 2014). Sin embargo, en este estudio la presencia del ecotipo oceánico fue en lugares con bajos relieves $\left(\overline{\mathrm{X}}=6.96^{\circ}\right)$, pero la mayor densidad de avistamientos se tuvo por encima del bajo de la isla San Pedro Nolasco (Guevara-Aguirre y Gallo-Reynoso, 2015), posiblemente aprovechando la alta concentración de clorofila que es ocasionada por este bajo profundo (Pérez y Sosa, 2014).

El presente estudio sugiere que el ecotipo costero en el golfo de California generalmente se alimenta y transita por la mañana, con socialización al mediodía y descansa por la tarde. El ecotipo oceánico se alimenta temprano y por la noche, socializa al mediodía, descansa por la tarde y transita por la mañana y la tarde.

En ambos ecotipos de toninas, los patrones de comportamiento resultaron en conformidad con la presencia de las diferentes variables estudiadas en cada área, lo que indica que cada ecotipo de tonina es dependiente de su hábitat local. Por lo tanto, se comprueba la hipótesis de hábitat local, ya que cada ecotipo depende del conjunto de las variables presentes en su hábitat. 


\section{Agradecimientos}

Este trabajo no hubiera sido posible sin el financiamiento de Comunidad y Biodiversidad (Cobi, A.C.) y Conacyt por la beca otorgada a DGA. Queremos expresar nuestra gratitud a todos los miembros del laboratorio de Ecofisiología por su valiosa contribución durante los recorridos, su gran amistad y cariño. También queremos agradecer a los Posgrados de Ciencias del Mar y Limnología, UNAM y al CIAD, A.C. por el espacio para poder analizar los datos. Finalmente, agradecemos a J.L. Higuera de las Guásimas y a J.L. Ramírez de La Manga por su valioso apoyo en el campo y su amistad.

\section{Referencias}

Aguirre, R. (2002). Los mares mexicanos a través de la percepción remota. México D.F.: Plaza y Valdéz.

Álvarez-Borrego, S. (2002). Physical scene: physical oceanography. En T. J. Case, M. L. Cody, y E. Escurra (Eds.), Island Biogeography in the Sea of Cortez (pp. 41-59). London: Oxford University Press.

Ballance, L. T. (1992). Habitat use patterns and ranges of the bottlenose dolphin in the Gulf of California, México. Marine Mammal Science, 8, 262-274.

Barros, N. B., Parsons, E. C. M. y Jefferson, T. H. (2000). Prey of offshore bottlenose dolphins from the South China Sea. Aquatic Mammals, 2-6, 26.1.

Baumgartner, M. F., Mullin, K. D., May, L. N. y Leming, T. D. (2001). Cetacean habitats in the northern Gulf of México. Fishery Bulletin, 99, 219-239.

Bazúa-Durán, C. y Herrera-Hernández, A. (2007). Bottlenose dolphin (Tursiops truncatus) phonations: how recording and usage are related. Bio-Acoustics, 29, 43-46.

Connor, R. C., Well, R. S., Mann, J. y Read, A. J. (2000). The bottlenose dolphin social relationships: in a fission-fusion society. En J. Mann, R. C. Connor, P. L. Tyack, y H. Whitehead (Eds.), Cetacean societies: field studies of whales and dolphins (pp. 91-126). Chicago: The University of Chicago Press.

Cubero, P. P. (2007). Distribución y condiciones ambientales asociadas al comportamiento del delfín bufeo (Tursiops truncatus) y el delfín manchado (Stenella attenuata) (Cetacea: Delphinidae). Revista de Biología Tropical, 55, 549-557.

De la Lanza-Espino, G. (1991). Oceanografía de mares mexicanos. México, D.F.: AGT Editores, S.A.

Díaz-Gamboa, R. E. (2004). Diferenciación entre tursiones Tursiops truncatus costeros y oceánicos en el golfo de California por medio de isótopos estables de carbono y nitrógeno Tesis de maestría. La Paz, Baja California Sur, México: Centro Interdisciplinario de Ciencias Marinas.

Eierman, L. E. y Connor, R. C. (2014). Foraging behavior, prey distribution, and microhabitat use by bottlenose dolphins Tursiops truncatus in a tropical atoll. Marine Ecology Progress Series, 503, 279-288.

Ferrero, R. C., Hobbies, R. C. y van Blaricom, G. R. (2002). Indicators of habitat use patterns among small cetaceans in the central North Pacific based on fisheries observer data. Journal of Cetacean Research and Management, 4 , 311-321.

Gallo-Reynoso, J. P. (1984). Interacción de calderones (Globicephala macrorhynchus) con la ballena de aleta (Balaenoptera physalus) y con tursiones (Tursiops truncatus gillii), (Mammalia: Cetacea). Anales del Instituto de Biología, Serie Zoología, Universidad Nacional Autónoma de México, 55, 331-333.

Gallo-Reynoso, J. P., Égido-Villareal, J. y Coria-Galindo, E. M. (2009). Sperm whale distribution and diving behavior in relation to presence of jumbo squid in Guaymas Basin, México. Marine Biodiversity Records, 2, 139-143.

Gallo-Reynoso, J. P., Égido-Villareal, J., Coria-Galindo, E. y Saldaña, J. (2006). Distribución, área de acción y población de toninas (Tursiops truncatus) en el área de la Bahía de las Guásimas, Sonora, México. Reporte técnico. Guaymas, Sonora: Centro de Investigación en Alimentación y Desarrollo, A.C. (CIAD).

Gendron, D. (2000). Familia Physeteridae. En S. T. Álvarez-Castañeda y J. L. Patton (Eds.), Mamíferos del noroeste de México (pp. 635-637). La Paz, Baja California Sur, México: Centro de Investigaciones Biológicas del Noroeste.
Guerrero-Ruiz, M. J., Urbán-Ramírez, J. y Rojas-Bracho, L. (2006). Las ballenas del golfo de California. Reporte técnico. México, D.F.: Semarnat and INE.

Guevara-Aguirre, D. (2011). Relación de la presencia de toninas (Tursiops truncatus) en la laguna de Términos, Campeche, México con los factores ambientales Tesis de maestría. México, D.F.: Posgrado de Ciencias del Mar y Limnología, Universidad Nacional Autónoma de México.

Guevara-Aguirre, D. y Gallo-Reynoso, J. P. (2015). Abundancia relativa y estacionalidad de dos poblaciones de toninas (Tursiops truncatus) en la región de Guaymas, Sonora, en el golfo de California. Therya, 6, 315-328.

Hoelzel, A. R. (1998). Genetic structure of Cetacean populations in sympatry, parapatry, and mixed assemblages: implications for conservation policy. Journal of Heredity, 89, 451-458.

Jaquet, N. y Gendron, D. (2002). Distribution and relative abundance of sperm whales in relation to key environmental features, squid landings and the distribution of other cetacean species in the Gulf of California, México. Marine Biology, 141, 591-601.

Klatsky, L. J., Wells, R. S. y Sweeney, J. C. (2007). Offshore bottlenose dolphins (Tursiops truncatus): movement and dive behavior near the Bermuda Pedestal. Journal of Mammalogy, 88, 599-666.

Lugo, H. J. (1986). Morfoestructuras del fondo oceánico mexicano. Boletín Instituto de Geografía, Universidad Nacional Autónoma de México, 15, 9-39.

Marcín-Medina, R. (2010). Uso del hábitat del tursión (Tursiops truncatus) en relación a factores ambientales y antropogénicos en la ensenada y sur de la bahía de La Paz, B.C.S, México. Tesis doctoral. La Paz, México: Centro Interdisciplinario de Ciencias Marinas, IPN.

Martínez-Serrano, I., Serrano, A., Heckel, G. y Schramm, Y. (2011). Distribución y ámbito hogareño de toninas (Tursiops truncatus) en Veracruz. México. Ciencias Marinas, 37, 379-392.

Medrano, G. L. y Urbán, R. J. (2002). La ballena jorobada (Megaptera novaeangliae) en la Norma Oficial Mexicana 059-ECOL-1994, 2000 [consultado 7 Ene 2016]. Disponible en: http://www.bionica/info/Biblioteca/Medrano2002.pdf.

Morteo, E., Heckel, G., Defran, R. H. y Schramm, Y. (2004). Distribution, movements and group size of the bottlenose dolphin (Tursiops truncatus) to the south of Santa Quintín Bay, Baja California, México. Ciencias Marinas, 30, $35-46$.

Natoli, A., Birkun, A., Aguilar, A., López, A. y Hoelzel, A. R. (2005). Habitat structure and the dispersal of male and female Bottlenose dolphins (Tursiops truncatus). Proceedings of the Royal Society, 272, 1217-1226.

Patiño-Valencia, J. L., Vargas, A. G. y Díaz, C. (2008). Estimación poblacional de toninas Tursiops truncatus en la bahía de Agiabampo Sonora-Sinaloa, México, en verano y otoño de 1995 al 2001. Revista de Zoología, 9, $15-21$.

Pérez-Arvizu, E. M., Aragón-Noriega, E. A. y Espinosa-Carreón, T. L. (2013). Variabilidad estacional de la clorofila a y su respuesta a condiciones El Niño y La Niña en el norte del golfo de California. Revista de Biología Marina y Oceanografía, 48, 131-141.

Pérez y Sosa, M. C. (2014). Distribución espacial de mamíferos marinos asociada a la geomorfología del fondo marino alrededor de la isla San Pedro Nolasco en el golfo de California Tesis de maestría. México, D.F: Universidad Nacional Autónoma de México.

Reza-García, N. I. (2001). Distribución y abundancia de Tursiops truncatus en la bahía de Santa María, Sinaloa, México Tesis. México, D.F.: Universidad Nacional Autónoma de México.

Roden, G. I. (1964). Oceanographic aspects of the Gulf of California. En T. H. Van Andel y G. G. Shor (Eds.), Marine Geology in the Gulf California, Memories American Petroleum Geologists, 3. Tulsa, Oklahoma: AAPG.

Santamaría del Ángel, E. y Álvarez-Borrego, S. (1994). Gulf of California biogeographic regions based on coastal zone color scanner imagery. Journal of Geophysical Research, 99, 7411-7421.

Segura, G. I. (2004). Diferenciación de ecotipos y estructura genética del delfín Tursiops truncatus en el golfo de California Tesis de maestría. Baja California, México: Centro de Investigación Científica y de Educación Superior de Ensenada, Ensenada.

Segura, G. I. (2011). Population genetics of species on the genera Tursiops and Delphinus within the Gulf of California and along the western coast of Baja California Tesis doctoral. Inglaterra: Universidad de Durham. 
Soberón, J. y Nakamura, M. (2009). Niches and distributional areas: concepts, methods and assumptions. Proceedings of the National Academy of Sciences, EE.UU., 106, 19644-19650.

Suárez-Esteban A., Miján I. (2011). Orca-Orcinus orca. Enciclopedia Virtual de los Vertebrados Españoles. Salvador, A., Cassinello, J. (Eds.). Museo Nacional de Ciencias Naturales, Madrid [consultado 5 May 2016]. Disponible en: http://www.vertebradosibericos.org/.

Tremblay, N. (2008). Variación estacional de los indicadores de estrés oxidativo asociada a la migración vertical de los eufáusidos subtropicales del golfo de California Tesis de maestría. La Paz, Baja California México: Centro Interdisciplinario de Ciencias Marinas, IPN.

Vázquez-Castán, L., Serrano, A. y Galindo, J. A. (2009). Estudio preliminar sobre la biodiversidad y abundancia de cetáceos en aguas profundas del golfo de México. Revista UDO Agrícola, 9, 992-997.

Wells, R. y Scott, M. (2002). Bottlenose dolphins Tursiops truncatus and T. aduncus. En W. Perrin, B. Würsig, y J. Thewiss (Eds.), Encyclopedia of Marine Mammals (p. 1414). San Diego: Academic Press. 